\title{
Dynamical Properties of Decorated Lamellar Microemulsions in the Brush Regime
}

\author{
By O. Holderer ${ }^{1, *}$, H. Frielinghaus ${ }^{1}$, D. Byelov ${ }^{2}$, M. Monkenbusch ${ }^{2}$, \\ J. Allgaier ${ }^{2}$, and D. Richter ${ }^{1,2}$ \\ ${ }^{1}$ IFF, Jülich Centre f. Neutron Sciences at FRM II, Forschungszentrum Jülich GmbH, \\ Lichtenbergstr. 1, 85747 Garching, Germany \\ ${ }^{2}$ IFF, Forschungszentrum Jülich GmbH, Wilhelm-Johnen-Straße, 52428 Jülich, Germany
}

(Received March 17, 2009; accepted June 16, 2009)

\section{Lamellar Microemulsion / Brush / Mushroom / Neutron Scattering / Dynamics}

The elastic constants of bicontinuous microemulsions, consisting of water, oil (decane) and a surfactant, can be modified by the addition of diblock-copolymers (boosting effect). A similar decoration of the surfactant membrane is possible in the lamellar phase. At high diblock copolymer densities, in the brush regime, a change in the dependence of the bending rigidity on the molecular size and concentration of the diblock copolymer is predicted theoretically. In particular a very strong dependence of the bending rigidity on the radius of gyration of the diblock copolymer $\left(\kappa \propto R^{5}\right)$ is predicted. We have studied the structure and dynamics of lamellar microemulsions by small angle neutron scattering (SANS) and neutron spin echo spectroscopy (NSE) in the brush regime, with high diblock copolymer density. Decyl polyglycol ether $\left(\mathrm{C}_{10} \mathrm{E}_{4}\right)$ has been used as surfactant, the amphiphilic diblock-copolymers were polyethylenepropylene/polyethyleneoxide $\left(\mathrm{PEP}_{5}-\mathrm{PEO}_{5}\right)$. The influence of the diblock copolymer concentration when entering the brush regime on the bending rigidity $\kappa$ and the correlation length $\xi$ will be discussed.

\section{Introduction}

Water, oil and a surfactant can form thermodynamically stable microemulsion phases [1-3]. A monolayer of surfactant molecules separates the water and oil phase. Lamellar phases of microemulsions are generated under certain conditions of temperature and surfactant concentration [4] in samples containing the same amount of water and oil. The surfactant efficiency in microemulsions can be largely enhanced upon addition of a small amount of diblock copolymers $[5,6]$.

\footnotetext{
* Corresponding author. E-mail: o.holderer@fz-juelich.de
} 
The copolymers are attached to the membrane, with its hydrophilic part extending into the water and the hydrophobic part into the oil. The diblock copolymer density was small enough that they stayed in the mushroom regime, i.e. the average distance between two polymer grafting points on the membrane is bigger than the end to end distance of the single blocks. The dynamics of such microemulsions in the lamellar phase is studied e.g. in [7]. Increasing the concentration of the amphiphilic block-copolymer (PEP5-PEO5) leads to a change in the dependence of the bending rigidiy on the molecular size and concentration $[8,9]$. Here, the dynamics of lamellar microemulsions decorated with diblock copolymers in the brush regime is studied.

\section{Samples}

The samples were a symmetric (1:1) mixture of deuterated water and deuterated decane plus the surfactant decyl polyglycol ether $\left(\mathrm{C}_{10} \mathrm{E}_{4}\right)$. Water and oil layers are separated by monolayers of the surfactant. In neutron scattering, the contrast between the protonated surfactant film and the deuterated surrounding medium is visible. In bicontinuous microemulsions, such mixtures form sponge-like structures with interpenetrating areas of oil and water. At higher surfactant concentrations, lamellar structures are obtained, where large almost flat membrane patches are stacked. If the lamellar domains can be oriented, a highly anisotropic scatternig is observed in SANS with "Bragg" peaks corresponding to the interlamellar distance. The surfactant membrane has been modified with the diblock copolymer polyethylenepropylene/polyethyleneoxide $\left(\mathrm{PEP}_{5}-\mathrm{PEO}_{5}\right)$ with a molecular weight of $5 \mathrm{~kg} / \mathrm{mol}$ for each of the two blocks. Similar to the surfactant, it has a hydrophilic part (PEO) and a hydrophobic part (PEP) and integrates into the surfactant membrane. The temperature range of the lamellar phase has been determined for each sample by observing the ability of birefringance of the sample with two polarizers. The mass fraction of the membrane, $\gamma$, has been kept almost constant for the samples doped with diblock copolymers. A three times higher surfactant contents has been used for the pure microemulsion, since the lamellar phase is shifted towards much higher surfactant contents in this case. The fraction of diblock copolymers in the membrane, $\delta$, has been varied from 0.12 to 0.32 . While the grafting density for the first sample is still low enough that the polymer coils in solution are separated and in the mushroom regime, it just enters the brush regime for $\delta=0.22$ and is well in the brush regime with overlapping polymer coils for the highest concentration.

\section{Neutron scattering}

The neutron scattering experiments have been performed at the FRJ2-Research Reactor of the Forschungszentrum Jülich. We performed neutron-spin-echo 
Table 1. Sample compositions.

\begin{tabular}{llllll}
\hline Sample & $\gamma$ & $\delta$ & $\mathrm{d}_{\text {domain }}[\AA]$ & $\mathrm{T}\left({ }^{\circ} \mathrm{C}\right)$ & Polymer regime \\
\hline 1 & 0.21 & 0 & 120 & 25.5 & - \\
2 & 0.065 & 0.12 & 540 & 29.8 & mushroom \\
3 & 0.070 & 0.22 & 540 & 32.9 & brush \\
4 & 0.075 & 0.32 & 540 & 30.0 & brush \\
\hline
\end{tabular}

(NSE) experiments [10] at the NSE-Spectrometer NSE-FRJ2 [11] and the small angle scattering (SANS) experiments at the SANS-facility KWS1-FRJ2. Especially for the NSE experiments with the long measuring times, slow fluctuations of large regions of the lamellar microemulsion have to be avoided. Therefore special sample cells have been used which contained an array of quartz lamellae, $0.2 \mathrm{~mm}$ thick with a spacing of $0.2 \mathrm{~mm}$ between the lamellae. When injected into the cell, the lamellar microemulsion is oriented with the surfactant membranes parallel to the quartz surfaces. The thickness of the cells was $2 \mathrm{~mm}$. In both experiments, the temperature of the sample has been controlled with thermostats and adjusted so that the samples were in the lamellar one-phase region of the tail of the fishtail phasediagram, exactly in the middle between upper and lower phase boundary temperature. The NSE experiments have been performed with q-values ranging from $0.05 \AA^{-1}$ to $0.28 \AA^{-1}$. Since the lamellar microemulsions scatter neutrons relatively good, quite large scattering angles are accessible.

\subsection{SANS experiments}

The scattering from lamellar microemulsions has been described by the Caillé theory [12]. Ordered lamellar microemulsions in film contrast show a peak at $q_{0}=2 \pi / d_{\text {layer }}$ where $d_{\text {layer }}$ is the distance between adjacent surfactant film layers. For well oriented samples, it is possible to obtain the bending rigidity and the compression modulus from fits of the peak. The scattering intensity from the four samples studied here is presented in Fig. 1. The pure microemulsion with a rather high surfactant contents is well oriented with clearly visible peaks at scattering vectors $q=2 \pi / d$ corresponding to the inter surfactant layer distance $\mathrm{d}_{\text {layer }}=58 \AA$. The smaller peaks at very low $\mathrm{q}$ are residual scattering from contrast differences between oil and water layers with $\mathrm{d}_{\text {domain }} \cong 2 \mathrm{~d}_{\text {layer }}$. The sample with $12 \%$ diblock-copolymer contents was not ordered enough to see the peak of the interlamellar distance. A very broad peak is present at very low q corresponding to the interdomain distance $d_{\text {domain }}=540 \AA$. With increasing diblock-copolymer contents the ordering of the lamellar phase increases and the Bragg peaks corresponding to the spacing of $d_{\text {layer }}=2 \pi / q_{0}=270 \AA$ between surfactant layers emerge. The sample with the highest diblock-copolymer concentration is nicely ordered as can be seen from the clear Bragg peaks. Still a determination of the Caille parameter has been impossible, for that the ordering in these relatively dilute lamellar phases was not good enough. The sample with $22 \%$ diblock-copolymer concentration turned out to be misaligned, which re- 

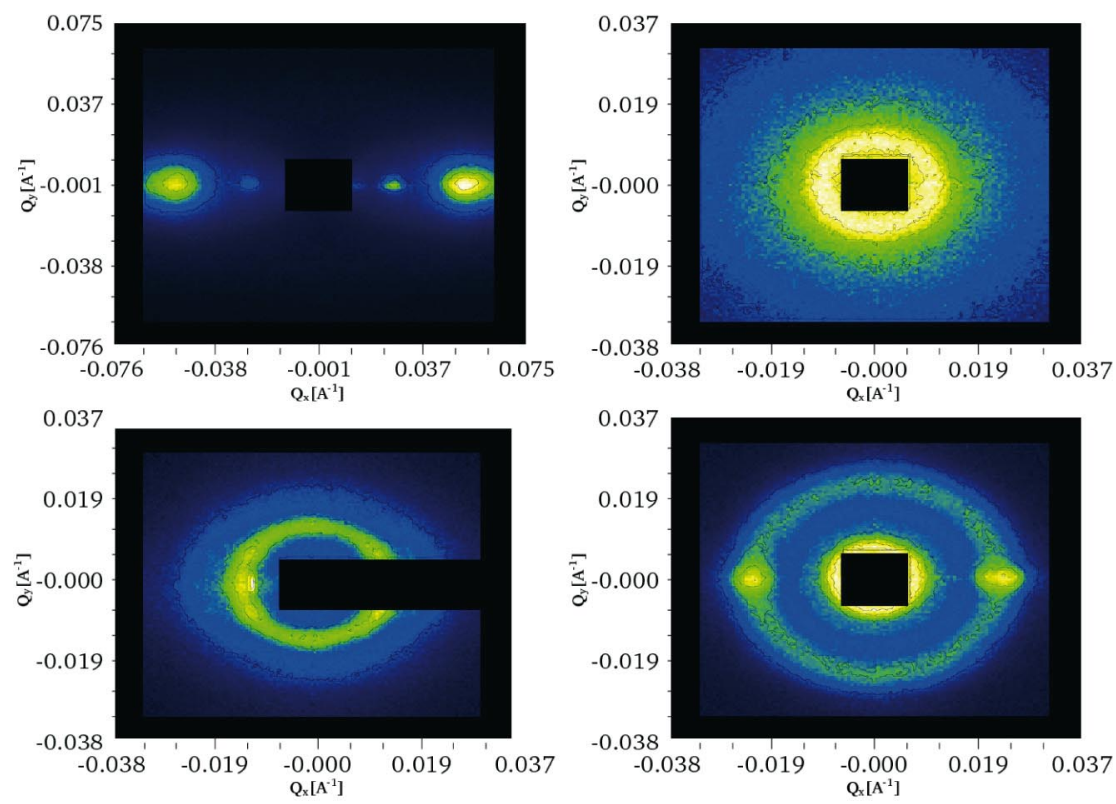

Fig. 1. Small angle scattering data from lamellar microemulsions.Top left: pure lamellar microemulsion, well oriented in the lamellar cell. Lamellar microemulsions with different polymer contents: $12 \%$ (top right), $22 \%$ (bottom left) and $32 \%$ (bottom right). The orientation is best for the sample with the highest diblock copolymer contents.

sulted in a strong peak of the beam totally reflected at the lamellar quartz plates. It is therefore also not possible to assess the grade of orientation in this sample. For the undulation mode spectrum measured with NSE, the lateral correlation length $\xi$ is the parameter determining the longest possible undulation waves. It is expected to scale with the bending rigidity and the inter-domain distance $\mathrm{d}$ approximately as $\xi \propto d \times \sqrt{\kappa}$. It can thus be concluded from the good ordering of the sample with the highest diblock-copolymer concentration that $\xi$ increases with increasing polmyer contents.

\subsection{Membrane dynamics measured with NSE}

The dynamics of the membrane is probed with neutron spin-echo spectroscopy. The intermediate scattering function $\mathrm{S}(\mathrm{q}, \mathrm{t})$ measures the timescale, on which the membranes fluctuate at the lengthscale determined by the q-vector. Figures 2 and 3 show the intermediate scattering function for the pure lamellar microemulsion and for the microemulsion with the highest polymer concentration. The 


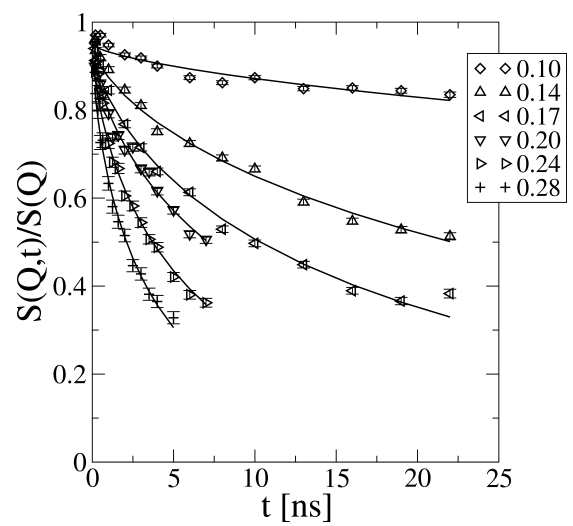

Fig. 2. Intermediate scattering function of the pure lamellar microemulsion for $\mathrm{q}$ ranging from 0.10 to $0.28 \AA^{-1}$.

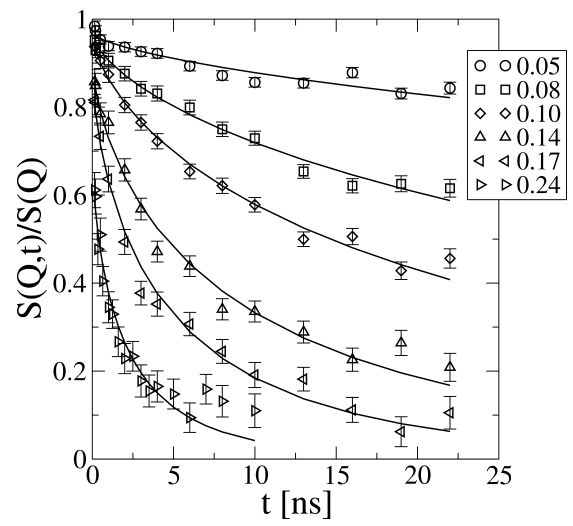

Fig. 3. Intermediate scattering function of a lamellar microemulsion in the brush regime with a diblock copolymer contents $\delta=0.32$ in the surfactant membrane for q ranging from 0.05 to $0.24 \AA^{-1}$.

intermediate scattering function is fitted with a stretchet exponential function according to the model described by Zilman and Granek [13].

$$
S(q, t) \simeq S(q) \exp \left(-\left(\Gamma_{q} t\right)\right)^{\beta}
$$

with $\beta=2 / 3$ and the $\mathrm{q}$-dependent relaxation rate $\Gamma_{q}$ :

$$
\Gamma_{q} \propto\left(\frac{k_{B} T}{\kappa}\right)^{1 / 2} \frac{k_{B} T}{\eta} q^{3}
$$

where $\kappa$ is the bending elasticity modulus and $\eta$ the solvent viscosity (we take the average viscosity of water and oil as the effective viscosity of the medium surrounding the membrane). It is conveniant to compare the average reduced 

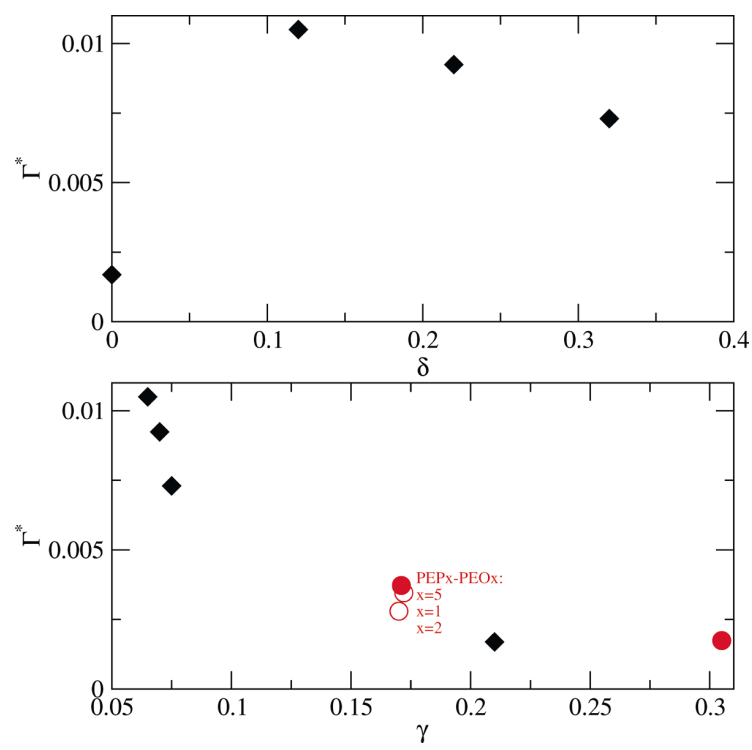

Fig. 4. Reduced relaxation rate as a function of polymer contents $\delta$ in the membrane (top) and as a function of the membrane mass fraction $\gamma$ in the sample (diamonds). The circles mark measurements taken from Ref. [7].

relaxation rate $\Gamma^{*}=\left\langle\left(\Gamma_{q} / q^{3}\right)\left(\eta(T) / k_{B} T\right)\right\rangle_{q>4 q 0}$, where viscosity effects and the qdependence have been eliminated. Fig. 4 shows $\Gamma^{*}$ as a function of $\delta$ and $\gamma$. Its dependence on $\gamma$ is stronger than for lamellar microemulsions in the mushroom regime. $\Gamma^{*}$ decreases slightly with $\delta$ which corresponds to an increase of the bending rigidity according to Equation 1. Since some approximations of Eqation 1 only hold for large $\kappa$ which is not true for the microemulsions under study, it has to be replaced by numerical integrations[7] over the undulation mode spectrum of the membrane patches to get the correct numerical value for the bending rigitidy. A graph linking the reduced relaxation rate of the evaluation with Eqation 1 to $\xi$ and $\kappa$ shown in Fig. 6. This procedure is described in Ref. [14]. The complete theory as well as the stretched exponential approximation requires that $q>>q_{0}$, a value of $q>4 q_{0}$ has proven to be adequate for applying the theoretical high-q models. For the analysis, the relaxation rate at the appropriate large q-values has been used. The measured reduced relaxation rates are plotted as bold lines in this representation. Since in these relatively dilute lamellar microemulsions the SANS scattering patterns were not usable for determining quantitatively the correlation length $\xi$, it is not possible to read the value of $\kappa$ from these plots. Here, another path is followed. The change of the bending rigidity is calculated for the two models, brush or mushroom regime of the sample, as shown in Fig. 5. It is thereby assumed that the bending rigidity is modified by the diblock copolymer in the case of the mushroom regime as 


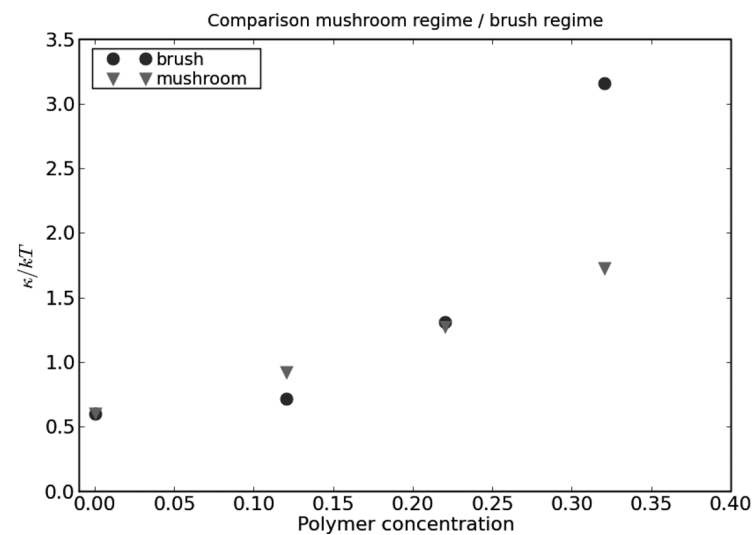

Fig. 5. Expected change of the bending rigidity $\kappa$ with polymer contents $\delta$ in the membrane. Both scenarios, assuming mushroom regime throuout and brush regime throughout are displayed.

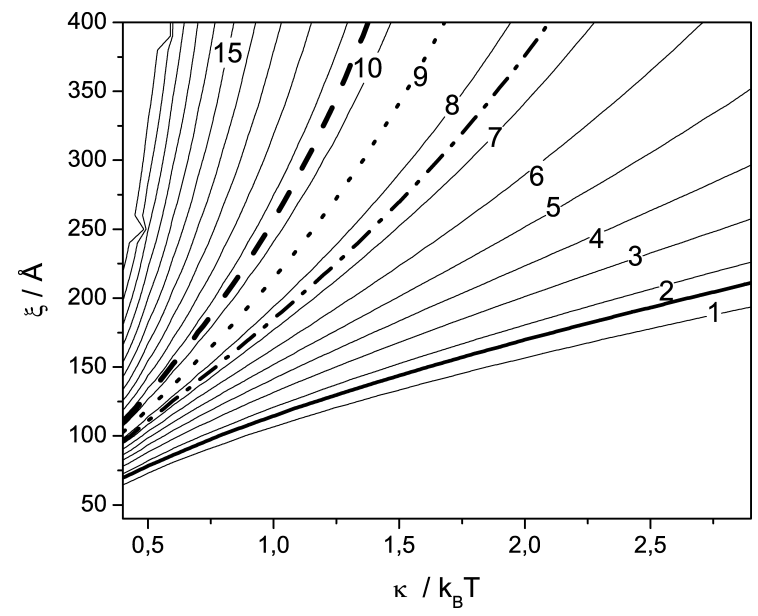

Fig. 6. Relaxation rate dependence on the bending rigidity $\kappa$ and the correlation length $\xi$. The contour lines represent the relaxation rate $\Gamma^{*} \times 1000$. The thick lines represent the relaxation rates of the four samples: solid: pure lamellar microemulsion; dashed: $\delta=0.12$, dotted: $\delta=$ 0.22 , dashed-dotted: $\delta=0.32$.

$$
\kappa_{e f f}=\kappa_{0}+\Xi l^{-2}\left(R_{o}^{2}+R_{w}^{2}\right)
$$

with the surface area per polymer $l^{2}$ and the end-to-end distance $R_{o}, R_{w}$ of the polymer in the oil and water phase respectively and a numerical factor $\Xi=$ 0.334 [15], whereas in the case of the brush regime a $R^{5}$-dependence is expected according to Ref. [8]: 


$$
\kappa_{e f f}=\kappa_{0}+\Xi l^{-5}\left(R_{o}^{5}+R_{w}^{5}\right)
$$

Using this as an input for the relation between $\Gamma, k$ and $\xi$-values from Fig. 6, $\xi$ is deduced. For the pure microemulsion, $\xi=90 \AA$ for $\kappa=0.6 \mathrm{k}_{B} \mathrm{~T}$. Using the mushroom model for all samples, one gets $\xi$-values which vary only very slightly between the different concentrations $(230,290$ and $300 \AA$ for the three values of $\delta=12,22$ and $32 \%$ respectively). The brush model gives very similar values for the lower two concentrations ( $180 \AA$ and $290 \AA$ ), the $32 \%$ sample leads to a correlation length of $700 \AA$, double the value as for the mushroom assumption.

\section{Conclusion}

Lamellar microemulsions with a high amount of amphiphilic diblock copolymers (PEP-PEO) have been prepared at relatively low membrane volume fractions of about $\delta=0.07$. The orientation has been largely improved by increasing the copolymer concentration from the mushroom into the brush regime, where the polymer coils overlap at the membrane surface and are stretched compared to the ideal gaussian conformation. Neutron spin-echo spectroscopy allowed to identify the pairs of $\xi$ and $\kappa$ corresponding to each concentration. Calculating the change of $\kappa$ in the two possible scenarios, that the change of the membrane elasticity follows either the prediction for the mushroom- or the brush-regime, allowed to get the corrsponding $\xi$ values. The mushroom prediction would result in only very small changes in $\xi$ which are not compatible with the largly improved orientation measured with SANS. It can be therefore concluded that for the sample with the highest diblock copolymer concentration the strong dependence of $\kappa$ on the radius of the diblock copolymer coils lead to a strong increase of $\kappa$ as predicted by theory [8] and that the correlation length is doubled compared to the sample in the mushroom regime.

\section{References}

1. M. Gradzielski, Curr. Opinion Coll. Interf. Sci. 13 (2008) 263.

2. G. Gompper and G. Schick, in Phase Transitions and Critical Phenomena. C. Domb and J. Lebowitz (Eds.), vol.16, Academic, London (1994).

3. T. Hellweg, Curr. Opinion Coll. Int. Sci. 7 (2002) 50.

4. M. Kahlweit, R. Strey, R. Schomaecker, and D. Haase, Langmuir 5 (1989) 305.

5. H. Endo, M. Mihailescu, M. Monkenbusch, J. Allgaier, G. Gompper, D. Richter, B. Jakobs, T. Sottmann, R. Strey, and I. Grillo, J. Chem. Phys. 1151 (2001) 580.

6. H. Endo, J. Allgaier, G. Gompper, B. Jakobs, M. Monkenbusch, D. Richter, T. Sottmann, and R. Strey, Phys. Rev. Lett. 851 (2000) 102.

7. M. Mihailescu, M. Monkenbusch, J. Allgaier, H. Frielinghaus, D. Richter, B. Jakobs, T. Sottmann, Phys. Rev. E 66 (2002) 041504.

8. C. Hiergeist and R. Lipowsky, J. Phys. II France 6 (1996) 1465.

9. K. Hristova, D. Needham, Macromolecules 28 (1995) 991.

10. F. Mezei, Proc. Workshop on Neutron Spin Echo. Springer:Berlin, (1980).

11. M. Monkenbusch, R. Schaetzler, and D. Richter, Nucl. Instrum. Methods Phys. Res. A A399 (1997) 301. 
12. M. A. Caillé, C. R. Seances, Acad. Sci., Ser. B 274 (1972) 1733.

13. A. Zilman, and R. Granek, Phys. Rev. Lett. 7723 (1996) 4788.

14. M. Monkenbusch, O. Holderer, H. Frielinghaus, D. Byelov, J. Allgaier, and D. Richter, J. Phys.: Cond. Mat. 17 (2005) S2903.

15. G. Gompper, H. Endo, M. Mihailescu, J. Allgaier, M. Monkenbusch, D. Richter, B. Jakobs, T. Sottmann, and R. Strey, Europhys. Lett. 565 (2001) 683. 
Bereitgestellt von | Forschungszentrum Jülich Angemeldet Heruntergeladen am | 24.04.18 16:01 